\title{
High-throughput heterodyne thermoreflectance: Application to thermal conductivity measurements of a Fe-Si-Ge thin film alloy library
}

\author{
Quentin d'Acremont, ${ }^{1,2, a)}$ Gilles Pernot, ${ }^{3}$ Jean-Michel Rampnoux, ${ }^{1}$ Andrej Furlan, ${ }^{4}$ \\ David Lacroix, ${ }^{3}$ Alfred Ludwig, ${ }^{4}$ and Stefan Dilhaire ${ }^{1}$ \\ ${ }^{1}$ Laboratoire Ondes et Matière d'Aquitaine (LOMA), UMR 5798, CNRS-Université de Bordeaux, \\ 33400 Talence, France \\ ${ }^{2}$ Amplitude-Systemes, 11 Avenue de la Canteranne Cité de la Photonique, 33600 Pessac, France \\ ${ }^{3}$ Laboratoire d'Energétique et de Mécanique Théorique et Appliquée (LEMTA), UMR 7563, CNRS-Université \\ de Lorraine, ENSEM, 2 Avenue de la Forêt de Haye, TSA 60604, 54518 Vandoeuvre-lès-Nancy Cedex, France \\ ${ }^{4}$ Institute for Materials, Ruhr-Universität Bochum, 44801 Bochum, Germany
}

\begin{abstract}
A High-Throughput Time-Domain ThermoReflectance (HT-TDTR) technique was developed to perform fast thermal conductivity measurements with minimum user actions required. This new setup is based on a heterodyne picosecond thermoreflectance system. The use of two different laser oscillators has been proven to reduce the acquisition time by two orders of magnitude and avoid the experimental artefacts usually induced by moving the elements present in TDTR systems. An amplitude modulation associated to a lock-in detection scheme is included to maintain a high sensitivity to thermal properties. We demonstrate the capabilities of the HT-TDTR setup to perform high-throughput thermal analysis by mapping thermal conductivity and interface resistances of a ternary thin film silicide library $\mathrm{Fe}_{x} \mathrm{Si}_{y} \mathrm{Ge}_{100} x_{y}$ ( $(20<x, y<80)$ deposited by wedge-type multi-layer method on a $100 \mathrm{~mm}$ diameter
\end{abstract}

\section{INTRODUCTION}

Time-domain thermoreflectance (TDTR) is a powerful technique allowing one to study ultrafast transient thermal transport. ${ }^{1,2}$ The first experiment using transient thermoreflectance was described in the late 1980s by Paddock and Eesley. ${ }^{3,4}$ Since then, it has been extensively used to measure thermal conductivities, ${ }^{5}$ heat capacities, ${ }^{5}$ and boundary thermal resistances. ${ }^{6}$ In addition, the method has been used to investigate ultrafast sub-picosecond mechanisms ${ }^{7}$ such as electron-electron ${ }^{8}$ and electron-phonon ${ }^{8,9}$ relaxations. The same setup has also been extensively used as a picosecond ultrasonic tool to measure thicknesses and acoustic velocities in thin layers. ${ }^{10}$

Practically, a transient thermoreflectance pump-probe setup is achieved by splitting an ultrashort laser pulse into a pump beam heating the sample and a probe beam measuring the temperature-induced reflectivity variations. A subpicosecond temporal resolution is obtained by varying the relative optical path length between the pump and probe beams with a mechanical delay stage. The pump beam is modulated in amplitude by an electro-optic modulator driven by a sine wave generator. Minute variations of the relative reflectivity signal $\left(\approx 10^{-6}\right)$ are obtained by filtering the measured modulated signal with a RF lock-in amplifier.

The achievable delay range to investigate thermal transport ranges from $1 \mathrm{ps}$ to $10 \mathrm{~ns}$, equivalent to a optical path difference of $300 \mu \mathrm{m}$ to $3 \mathrm{~m}$. For "long" pump-probe delays

\footnotetext{
a) Author to whom correspondence should be addressed: quentin.dacremont@ u-bordeaux.fr
}

(>few hundreds of ps), the mechanical delay stage may be a source of beam defocusing and misalignment. The interpretation of the lock-in signals is therefore not straightforward because of distorted signals due to those artefacts. A solution to this issue has been proposed: ${ }^{11}$ Instead of analyzing separately the in-phase $V_{\text {in }}$ and out-of-phase $V_{\text {out }}$ signals delivered by the lock-in amplifier, the ratio $-V_{\text {in }} / V_{\text {out }}$ overcomes these artefacts since both signals are similarly affected by defocusing or a change of the overlapping. It has also been shown ${ }^{12}$ that the ratio signal is highly sensitive to thermal conductivity and carries valuable information about the thermal transport.

The most recent improvement to the transient thermoreflectance technique is the Heterodyne Picosecond Thermoreflectance (HPTR) setup as described by Dilhaire et al. for nanoscale thermal identification in $2011^{13}$ and based on an Asynchronous Optical Sampling first described by Elzinga et al. in $1987 .{ }^{14}$ In this setup, pump and probe beams come from two separated but synchronized oscillators with repetition rates slightly shifted from each other. This frequency difference replaces the mechanical delay line, eliminating all moving parts from the setup and improving the accuracy of the measurements. In this case, variations of beam defocusing and overlapping are suppressed while the available time range is solely limited by the pump oscillator's period (>10 ns). It is worth noting that the acquisition speed is also much higher, since the time needed for a single acquisition is the inverse of the frequency shift $(\leq 1 \mathrm{~s})$, instead of being limited by the speed of the mechanical delay stage.

High-throughput measurement of thermal properties has been performed by Zheng et al. in 2007. ${ }^{15}$ It was based on a 


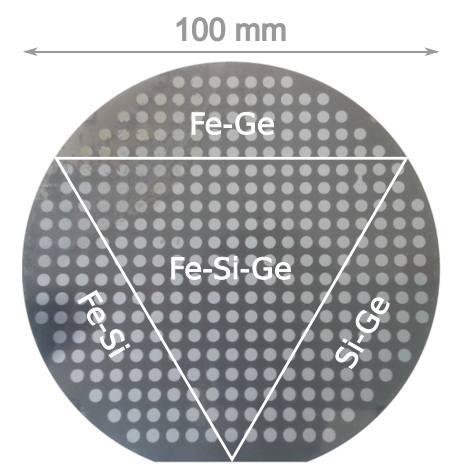

FIG. 1. Ternary Fe-Si-Ge alloy library deposited on a $100 \mathrm{~mm}$ sapphire wafer.

classical TDTR with a mechanical and limited delay line. To perform a large amount of measurements, the authors chose to use a single delay with a maximized sensitivity to thermal conductivity, then the acquisition speed being 3 points/s. Nevertheless, this approach can lead to numerous errors by assuming unknown thermal interface resistances to be constant and as discussed later, neglecting the post-acquisition adjustment of the phase of the lock-in amplifier.

In this paper, we present a high-throughput time-domain thermoreflectance (HT-TDTR) setup, which is an improved version of the HPTR setup, developed to carry out high-speed and high-throughput thermal conductivity measurements with minimum user actions required. This setup allows performing thermal conductivity mapping of large surface samples.

To demonstrate the efficiency of our setup, a ternary thin film silicide library $\mathrm{Fe}_{x} \mathrm{Si}_{y} \mathrm{Ge}_{100-x-y}$ (Fig. 1) has been deposited by wedge-type multi-layer method. This library comprises 342 measurement areas of different ternary compositions over a $100 \mathrm{~mm}$ diameter sapphire wafer. We report the mapping of the thermal conductivities of this Fe-Si-Ge library and consequently demonstrate the efficiency of our HTTDTR setup. We performed 33 measurements over the entire surface, representative for the complete material system. The capabilities and performances of our setup are discussed in the following.

\section{TIME-DOMAIN THERMOREFLECTANCE MEASUREMENTS}

\section{A. Thermoreflectance measurement principle and modeling}

Thermoreflectance techniques are based on an optical measurement of the temperature through the variations of the refractive index. In most materials, the refractive index variations come from both acoustic strains and temperature changes. The contribution of these two phenomena to the measured signal depends on the probe wavelength and on the nature of the metallic transducer deposited on the top of the layer of interest. Choosing an appropriate wavelength and metal, where the detected acoustic contribution is minimal, allows being sensitive almost only to the thermal transport. To fulfill those criteria, a probe wavelength at $810 \mathrm{~nm}$ and an aluminum transducer were chosen in this work. Hence, the relative variation of the reflectivity signal is proportional to the temperature variations in the metal film,

$$
\frac{\Delta R}{R} \propto \Delta \Theta_{\text {metal }} .
$$

Fitting the recorded signal with a Fourier transport thermal model, described by quadrupoles matrices, allows retrieving the thermal conductivity and the boundary thermal resistance of the layer of interest, assuming that all the other parameters of the sample (thermal conductivities, boundary thermal resistances, thicknesses, and volumetric heat capacities) are known. This Fourier transport model has been extensively described. ${ }^{13}$ Assuming a cylindrical symmetry and a multilayer structure, the heat flux in Laplace-Hankel domain in the layer $i$ is written as follows:

$$
\Phi_{i}\left(z, \alpha_{H}, s\right)=-\lambda_{i} q_{i} K_{i}^{1} \sinh \left(q_{i} z\right)-\lambda_{i} q_{i} K_{i}^{2} \cosh \left(q_{i} z\right),
$$

where $\lambda_{i}$ is the thermal conductivity of layer $i, K_{i}^{1}$ and $K_{i}^{2}$ are unknown constants, $s$ and $\alpha_{H}$ are, respectively, Fourier and Hankel variables, and $q_{i}$ is a function of the following variables:

$$
q_{i}^{2}=\alpha_{H}^{2}+\frac{\rho_{i} C_{i}}{k_{i}} s,
$$

where $\rho_{i}$ and $C_{i}$ being, respectively, the mass density and the specific heat capacity of layer $i$. This multi-equation system can be expressed by a quadrupole matrix,

$$
\left[\begin{array}{c}
\Theta_{\text {in }} \\
\Phi_{\text {in }}
\end{array}\right]=\left[\begin{array}{cc}
A_{i} & C_{i} \\
B_{i} & D_{i}
\end{array}\right]\left[\begin{array}{l}
\Theta_{\text {out }} \\
\Phi_{\text {out }}
\end{array}\right],
$$

where $\Phi_{\text {in }}$ and $\Phi_{\text {out }}$ are, respectively, the heat flows penetrating and exiting the sample. $\Theta_{\text {in }}$ and $\Theta_{\text {out }}$ are, respectively, the top and bottom surface temperatures. The product matrix $\left[\begin{array}{ll}A_{i} & B_{i} \\ C_{i} & D_{i}\end{array}\right]$ is determined by multiplying descriptive matrices of each layer and thermal boundary resistances. The coefficients depend on the thickness of layer $i$ as well as its thermal properties. Inverse numerical transforms of expression 4 gives the temperature solution in space and time domains. Using Eq. (1), the measured signal can be related to the calculated temperature. The last step relates the single pulse temperature to the effects of multiple pulse accumulation ${ }^{16}$ and lock-in detection. The acquired in-phase $V_{\text {in }}$ and out-of-phase $V_{\text {out }}$ signals can be expressed as

$$
\begin{gathered}
V_{\text {in }}=A_{\text {in }} \sum_{k=-\infty}^{\infty} \sum_{q=-\infty}^{\infty} \Delta r(k \Delta T+q T) \cos \left(2 \pi f_{m}(k \Delta T+q T)\right), \\
V_{\text {out }}=A_{\text {out }} \sum_{k=-\infty}^{\infty} \sum_{q=-\infty}^{\infty} \Delta r(k \Delta T+q T) \sin \left(2 \pi f_{m}(k \Delta T+q T)\right) .
\end{gathered}
$$

$A_{\text {in }}$ and $A_{\text {out }}$ are constants depending on modulation amplitude and oscillators' powers and frequencies. $T$ and $\Delta T$ are, respectively, the pump oscillator period and the difference between the two oscillators' periods. $f_{m}$ is the modulation frequency of the pump beam.

\section{B. High-throughput thermoreflectance setup}

The HT-TDTR setup (Fig. 2) is based on two coherent Ti:Sa oscillators with a mean repetition rate of $76.2 \mathrm{MHz}$ and 


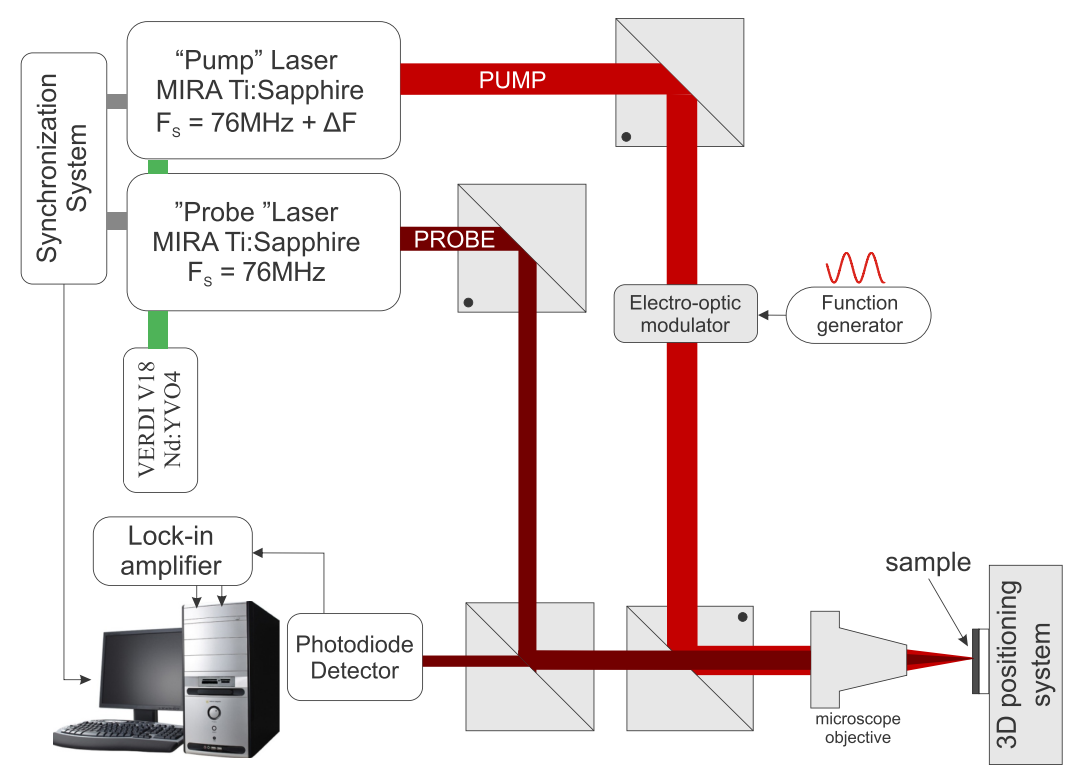

FIG. 2. High-throughput pump-probe thermoreflectance setup.

pumped by a continuous $18 \mathrm{~W}$-Verdi Nd:YVO4 at $532 \mathrm{~nm}$. Pump and probe beams' wavelengths have been set, respectively, at $780 \mathrm{~nm}$ and $810 \mathrm{~nm}$. The frequency shift between oscillators is set at $\Delta F=1 \mathrm{~Hz}$, which gives a single acquisition of a complete signal in $1 \mathrm{~s}$. The pump beam passes through an electro-optical modulator (ConOptics 350-160), driven by a $200 \mathrm{MHz}$ amplifier and a function generator (Agilent 33250A) at $9.76 \mathrm{MHz}$, which adds an amplitude modulation to the pulse comb. A lock-in amplifier (Stanford Research Systems SR844) time constant is set at $30 \mu \mathrm{s}$ for acquisition speed purposes. Pump and probe beams are recombined and focused on the sample with a $10 \times$ planar microscope objective. The pump and probe beams have a respective focused size of $9.5 \mu \mathrm{m}$ and $7 \mu \mathrm{m}$. The reflected signal is detected by a Thorlabs DET-10A silicon photodiode. The parameters of this setup are summarized in Table I and typical values of the same parameters are given for a delay-line/single oscillator thermoreflectance setup.

A precise comparison of the delay-line based and HTTDTR setups is difficult; the signal-to-noise ratio depends on various parameters such as the stability in power of the laser oscillators, the reflectivity signal of the sample, and the intrinsic electronic noise that is different from one experimental setup to another. For those reasons, Table I summarizes general values for acquisition speed and equivalent scanning frequency. A single acquisition of the entire delay takes approximately $80 \mathrm{~s}$ with a high-speed mechanical line $(500 \mathrm{~mm} / \mathrm{s})$. Using the HT-TDTR with a shift frequency of $1 \mathrm{~Hz}$, this time is reduced to $1 \mathrm{~s}$. Since multiple acquisitions (typically 10) are required for averaging purpose, $10 \mathrm{~s}$ is therefore needed with the HT-TDTR, while more than $10 \mathrm{~min}$ is usually required with the classical to obtain a reflectivity signal with an equivalent signal-to-noise ratio.

To scan the entire $100 \mathrm{~mm}$ diameter library as presented in Fig. 1, we have implemented a fully automated 3D positioning system made of three $150 \mathrm{~mm}$ motorized stages with a $100 \mathrm{~nm}$ step resolution. By recording an image of pump and probe focused on a camera, the stage along the beam's axis ensures an automatic focusing of the beams on the sample. Prior to any measurement, a $100 \mathrm{~nm}$ step scan over few micrometers is performed while beam sizes are recorded and then fitted, and the stage is accordingly moved to guarantee optimal focusing. This helps securing a high signalto-noise ratio as well as providing a constant size of the heat source. The knowledge of the beam sizes is necessary as they are input parameters for the $3 \mathrm{D}$ cylindrical heating model used to adjust the experimental data. The two other motorized stages are used to center the $\mathrm{Al}$ metallized measurement areas of the material library on the focused beams as well as automatically moving from one area to another.

TABLE I. Main parameters of the HT-TDTR setup and typical parameters for a simple delay line thermoreflectance setup. Both setups are assumed to have $76.2 \mathrm{MHz}$ oscillators and the same available temporal scan range of $13.123 \mathrm{~ns}$.

\begin{tabular}{lcc}
\hline \hline & $\begin{array}{c}\text { Delay-line, } \\
\text { single oscillator } \\
\text { Parameter }\end{array}$ & Hist-TDTR \\
\hline $\begin{array}{l}\text { Oscillator } \\
\text { wavelength }\end{array}$ & Visible - IR & $\begin{array}{c}\text { Double oscillator } \\
780 \mathrm{~nm} / 810 \mathrm{~nm}\end{array}$ \\
$\begin{array}{l}\text { Oscillator } \\
\text { frequency }\end{array}$ & $\begin{array}{c}76.2 \mathrm{MHz} \\
\text { Temporal }\end{array}$ & $76.200001 \mathrm{MHz}$ \\
scan range & $76.2 \mathrm{MHz}$ & $13.123 \mathrm{~ns}$ \\
$\begin{array}{l}\text { Max. delay } \\
\text { line length }\end{array}$ & $13.123 \mathrm{~ns}$ & None \\
$\begin{array}{l}\text { Time needed for } \\
\text { a single } \\
\text { acquisition }\end{array}$ & $3.94 \mathrm{~m}$ & $\begin{array}{c}\text { Inverse of the } \\
\text { frequency shift }\end{array}$ \\
$\begin{array}{l}\text { Acquisition } \\
\text { frequency }\end{array}$ & $\approx 80 \mathrm{~s}\left(50 \mathrm{~mm} \mathrm{~s}^{-1}\right.$ & between oscillators: $1 \mathrm{~s}$ \\
$\begin{array}{l}\text { Equivalent } \\
\text { mechanical } \\
\text { stage speed }\end{array}$ & stage $)$ & $1 \mathrm{~Hz}$ \\
\hline \hline
\end{tabular}




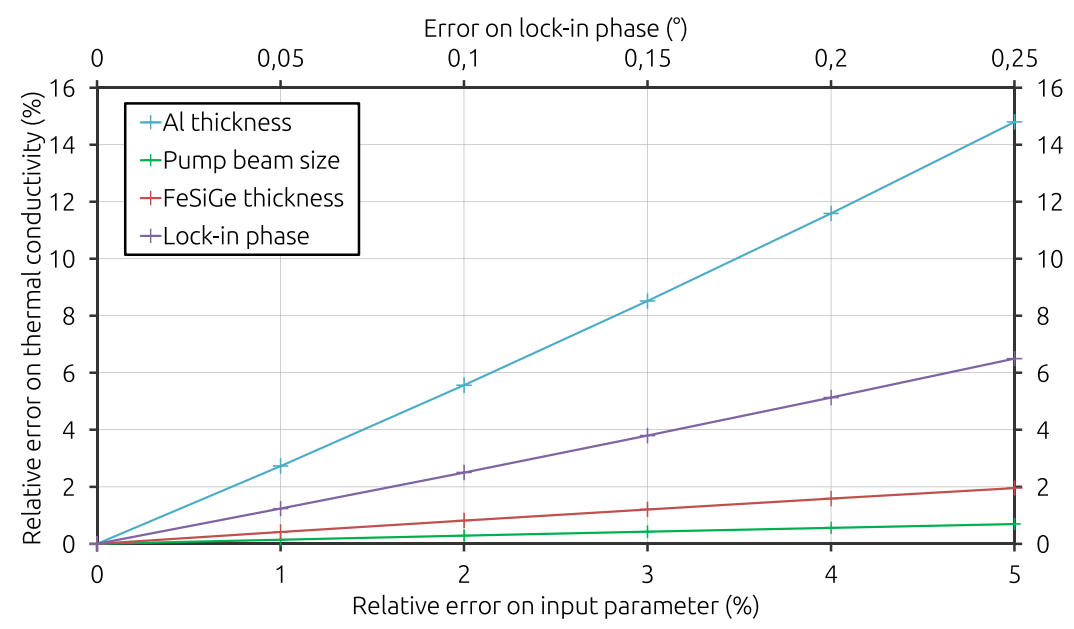

FIG. 3. Plot of the error on the identified thermal conductivity for a relative variation of four different input parameters for $\mathrm{Fe}_{70} \mathrm{Si}_{29} \mathrm{Ge}_{1}$. Blue (respectively, red, green, and purple) lines refer to the input $\mathrm{Al}$ thickness (respectively, Fe-Si-Ge thickness, pump beam size, and lock-in phase). Simulated parameter values are as follows: Al thickness: $70 \mathrm{~nm}$; Fe-Si-Ge thickness: $354 \mathrm{~nm}$; pump beam size: $9.5 \mu \mathrm{m}$.

Although the addition of this positioning system does not have any impact on a single area measurement, it significantly helps when dealing with a large amount of measurements performed during a high-throughput analysis of a material library. Figure 3 shows a plot of relative variation of the identified thermal conductivity for an error on various input parameters. As shown, a $5 \%$ relative error on a $9.5 \mu \mathrm{m}$ pump beam waist results in a $1 \%$ relative error on the identified thermal conductivity. Probe beam size errors are of the same order of magnitude.

The reliability of a measurement is assessed by several factors, including repeatability. When measuring a large number of samples on a material library, the repeatability over time is often an issue since the exact same positioning is almost impossible to achieve by moving manually a translation stage. In HT-TDTR measurements, every scanned position is recorded and stored for potential future use. In most cases, verifying the results by repeating the measurement is mandatory, and in our case, it can be achieved with high spatial precision.

Lock-in amplifier's phase adjustment is a critical factor in modulated TDTR experiments. When heated by a laser pulse, an almost instantaneous rise of temperature of the sample is observed on the real part of the signal $V_{i n}$, while the imaginary part $V_{\text {out }}$ remains almost constant around $\mathrm{t}=0$. As shown in Fig. 4, this can be seen in the Nyquist plane $V_{\text {out }}(t)=f\left(V_{\text {in }}(t)\right)$ as a horizontal line between negative and positive delay around zero $\left(V_{\text {out }}\left(t \rightarrow 0^{-}\right)=V_{\text {out }}\left(t \rightarrow 0^{+}\right)\right)$. Any additional phase caused by the electrical circuitry or the lock-in itself induces an angular error and therefore a mixing between real and imaginary signals. For every measurement, the phase on the lock-in amplifier is measured and automatically corrected to obtain a horizontal shaped curve around zero in the Nyquist plane. As seen in Fig. 3, a small $0.2^{\circ}$ error on phase adjustment leads to a $5 \%$ relative error on the identified thermal conductivity, highlighting the necessity of a precise lock-in phase correction.

A possible unintended variation of the $\mathrm{Al}$ thickness is also an important factor for thermal analysis. As presented in Fig. 4, a $5 \%$ relative error on a 70 nm-thick Al layer results in a major $15 \%$ relative error on thermal conductivity. The same comparison can also be done on the thickness of the film of interest: a $5 \%$ relative error on the $354 \mathrm{~nm}$-thick Fe-Si-Ge layer leads to a $2 \%$ relative error on the identified thermal conductivity. The HT-TDTR setup, used for picosecond ultrasonic measurements tool, may provide few nanometers precise measurements of thicknesses, assuming sound velocity in $\mathrm{Al}$ as known, which drastically reduces significant errors. With its efficient and precise spatial scanning, we can check the thickness variations over large samples and consequently avoid inaccuracies in the thermal analysis. In this case, picosecond ultrasonic is a powerful method to assess the validity of the deposition since fabrication processes rely on either thickness uniformity or precise linear gradients.

The identification of thermal properties (i.e., thermal conductivity and thermal boundary resistance) is automatically performed for every measurement once the acquisition is done. With the improvements discussed earlier, most of the user actions required between two consecutive measurements are automated and more precise, resulting in a net gain in both acquisition time and accuracy. More precisely, with a pre-processing time of $10 \mathrm{~s}$ for positioning and focusing, and 10 averaging at a frequency shift of $1 \mathrm{~Hz}$, the

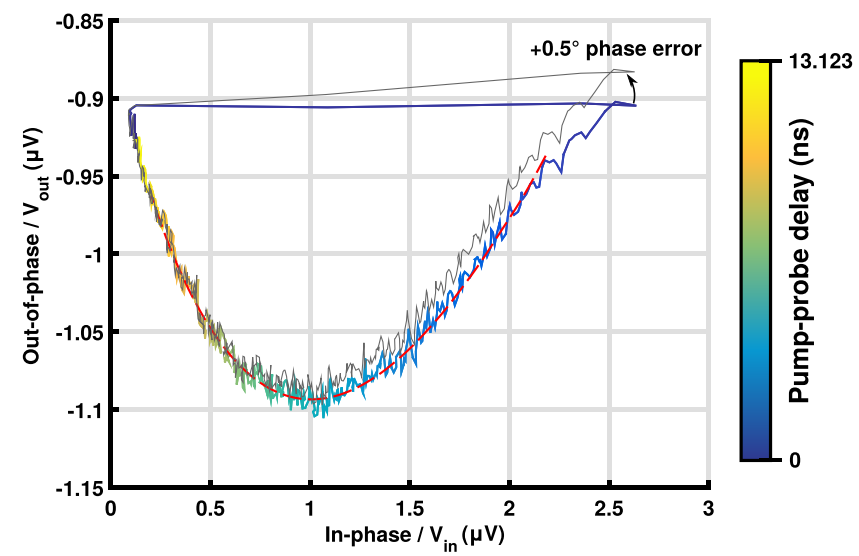

FIG. 4. Nyquist plot $V_{\text {out }}(t)=f\left(V_{\text {in }}(t)\right)$ for $\mathrm{Fe}_{36} \mathrm{Si}_{28} \mathrm{Ge}_{36}$. Solid line is the measured lock-in amplifier signal in the Nyquist plane and is colored according to time, from dark blue for a zero pump-probe delay to bright yellow for 13.123 ns. Dashed red line is the best fit obtained. Grey line is the measured lock-in amplifier signal with a $+0.5^{\circ}$ phase error. 
total measurement time for a single area is $20 \mathrm{~s}$, compared to roughly $15 \mathrm{~min}$ for a classical delay-line setup meaning an acquisition time reduction by a factor of 45 . In addition, the post-processing time is reduced from $5 \mathrm{~min}$ down to 2 min with the automated processing. Our novel HT-TDTR setup consequently allows a high-throughput characterization of thin film libraries, from measurements to thermal parameters identification. The automatic positioning system and lock-in amplifier phase adjustment guarantee the same measurement conditions (positions, beams sizes) for every scanned area.

\section{APPLICATION TO THERMAL CONDUCTIVITY MAPPING OF A Fe-Si-Ge THIN FILM LIBRARY}

Silicide alloys, including ternaries, have recently started to be interesting in terms of their thermoelectric properties. ${ }^{17-20}$ In addition, their abundance and low average costs make them excellent thermoelectric candidates. However, due to the complexity of the ternary materials, a wide range of compositions have to be studied to find optimal compositions that would maximize the thermoelectric figure of merit.

\section{A. Combinatorial synthesis by wedge-type multi-layer deposition method}

For the deposition of Fe-Si-Ge libraries, a combinatorial approach was taken (Fig. 5), which made it possible to achieve compositions from $0 \%$ to around $80 \%$ for all three elements. The used wedge-type multilayer approach was described previously. ${ }^{21,22}$ This method is suited for producing thin film libraries of a wide range of compositions. It produces simultaneously with the ternary library also three binary systems by the combinations of the three elements. It requires, though, long deposition times and high post-deposition annealing temperatures for alloy formation from the precursor multilayered structure. In the present study, the full range of up to $100 \%$ could not be achieved due to our priority to maximize the ternary composition area.

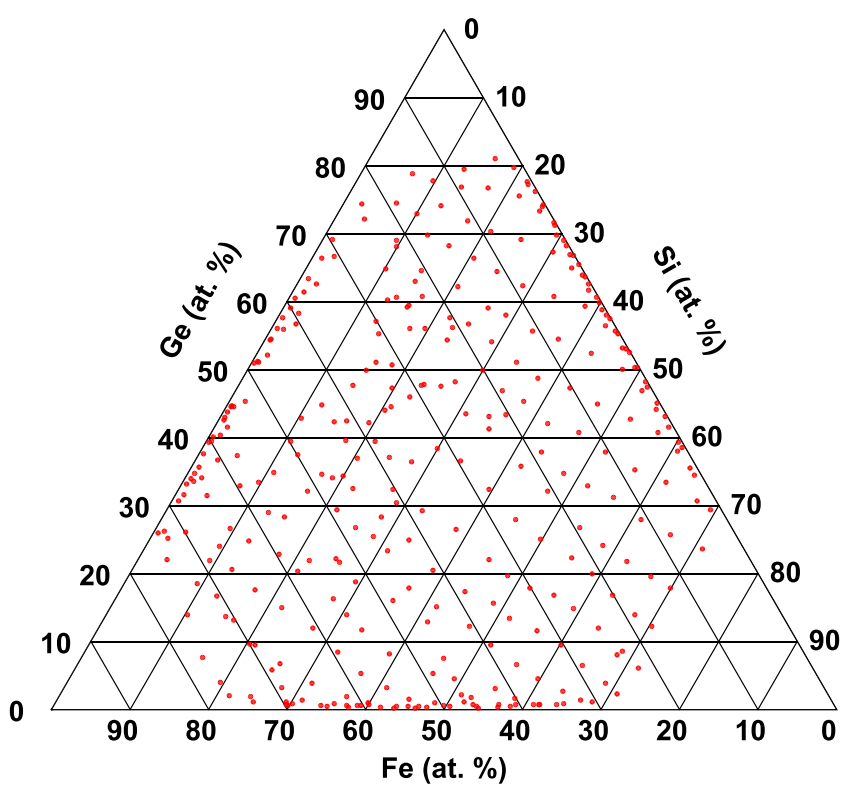

FIG. 6. Compositions in the Fe-Si-Ge thin film library as determined by highthroughput EDX measurements.

A Fe-Si-Ge film library was deposited on a singlecrystalline $100 \mathrm{~mm}$ diameter sapphire wafer. Single element layers were deposited from elemental targets of $\mathrm{Fe}(99.99 \%)$, $\mathrm{Si}(99.999 \%)$, and $\mathrm{Ge}(99.999 \%)$. Ar (6 N) was used as a process gas at $0.67 \mathrm{~Pa}$ pressure and $60 \mathrm{SCCM}$ flow. The process gave a multi-layer film with the single layer wedge thicknesses at the wafer center of around $1 \mathrm{~nm}$ for $\mathrm{Fe}, 1.7 \mathrm{~nm}$ for $\mathrm{Si}$, and $2 \mathrm{~nm}$ for Ge. In order to transform the multilayer thin film into a multiphase alloy, the Fe-Si-Ge library was annealed in $\mathrm{Ar}$ atmosphere at $800^{\circ} \mathrm{C}$ for $80 \mathrm{~h}$. Lower temperature, or shorter, annealing processes proved to be ineffective.

\section{B. Mapping thermal properties of $\mathrm{Fe}-\mathrm{Si}-\mathrm{Ge}$}

Thicknesses of the Fe-Si-Ge thin films for every measurement area have been obtained by an AMBIOS profilometer for the 342 areas across the wafer. For thickness measurements, a photoresist was applied on areas to be measured. Subsequent to film deposition, the photoresist was removed by
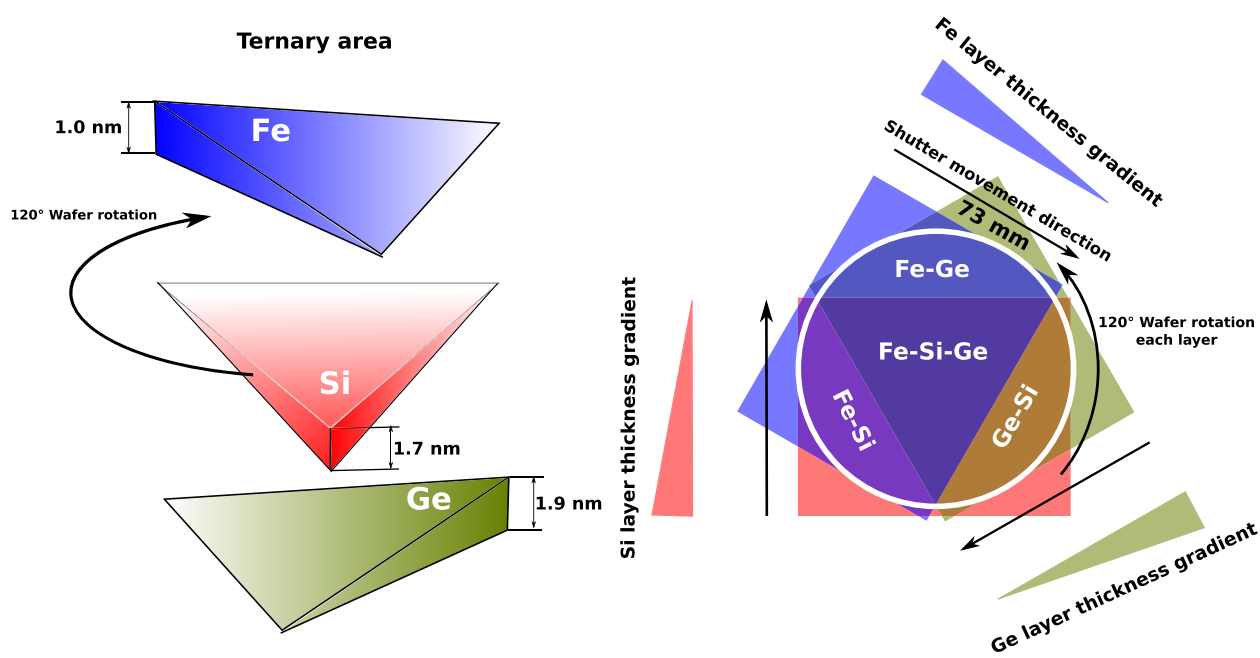

FIG. 5. Schematic of the wedge-type multi-layer deposition method. 
acetone and isopropanol, exposing the surface of the substrate and enabling film thickness measurement by determination of height between substrate and film surface. The examined FeSi-Ge library thickness was ranging from $200 \mathrm{~nm}$ to $900 \mathrm{~nm}$. A $70 \mathrm{~nm}$-thick Al transducer layer was deposited by electron beam evaporation on top of the Fe-Si-Ge library. Fe$\mathrm{Si}-\mathrm{Ge}$ library compositions for the analyzed areas have been determined by Energy dispersive X-ray spectroscopy (EDX) using an automated Oxford Instruments INCA X-act detector attached to a JEOL 5800LV scanning electron microscope (SEM) operating at $20 \mathrm{kV}$. Mapping was done for a mesh of 342 spots across TFL surface, of size $400 \mu \mathrm{m}$ $\times 600 \mu \mathrm{m}$ mutually separated by $4.5 \mathrm{~mm}$ and represented in Fig. 6 .

33 measurement points have been taken on this library, spread in a wide and representative range of compositions. Thermal conductivity and thermal boundary resistance measurements on Fe-Si-Ge are shown in Fig. 7.

Since HT-TDTR is mostly sensitive to the thermal effusivity, the volumetric heat capacity $\rho C$ of the Fe-Si-Ge alloy, for every composition, must be known. In our case, they have been deduced from a weighted average method taking the composition of each point of measurement, as determined by the EDX measurements showed earlier,
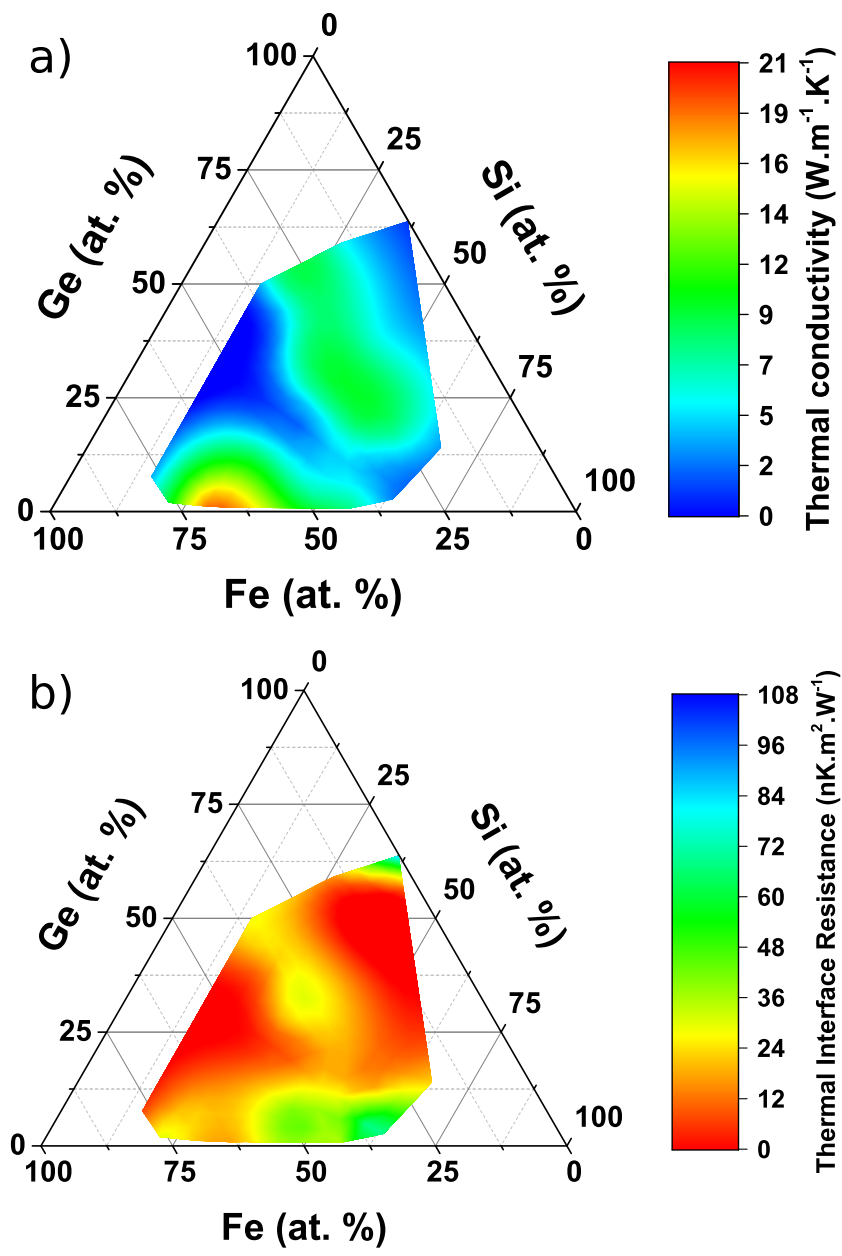

FIG. 7. (a) Ternary graph of measured thermal conductivities on a multiwedge deposited Fe-Si-Ge alloy library. (b) Ternary graph of the measured thermal interface resistances on a multi-wedge deposited Fe-Si-Ge alloy library.

$$
\begin{aligned}
(\rho C)_{\mathrm{Fe}_{x} \mathrm{Si}_{y} \mathrm{Ge}_{100-x-y}}= & \frac{x}{100} \times \rho_{\mathrm{Fe}} C_{\mathrm{Fe}}+\frac{y}{100} \times \rho_{\mathrm{Si}} C_{\mathrm{Si}} \\
& +\frac{100-x-y}{100} \times \rho_{\mathrm{Ge}} C_{\mathrm{Ge}} .
\end{aligned}
$$

The volumetric heat capacity of the $650 \mu \mathrm{m}$-thick sapphire substrate was assumed to have the classic literature value of $3.1 \times 10^{6} \mathrm{~J} \mathrm{~K}^{-1} \mathrm{~m}^{-3}$.

The Si-Ge-Fe library exhibits thermal conductivity values from $1.1 \mathrm{~W} \mathrm{~m}^{-1} \mathrm{~K}^{-1}$ for $\mathrm{Fe}_{38} \mathrm{Si}_{25} \mathrm{Ge}_{37}$ up to $20.7 \mathrm{~W} \mathrm{~m}^{-1} \mathrm{~K}^{-1}$ for $\mathrm{Fe}_{70} \mathrm{Si}_{29} \mathrm{Ge}_{1}$. Very low values of thermal conductivity are a clear indicator for amorphous phases of ternary alloys. This was confirmed by XRD analysis. Binary alloys (Fe 0\%) and ternary alloys close to Fe 50\% exhibit such amorphous limit thermal conductivity values. The amorphous behavior is also confirmed by the interface resistance map where no physical values were found on those specific areas. For a thick amorphous layer, very large thermal resistance leads to a reduction of the sensitivity of the TDTR method to identify the metal/layer interface resistance. For the same reasons, no HT-TDTR measurements were performed for Ge $0 \%$, where XRD analysis has revealed important amorphous phases. Crystalline phases were found close to the center of the ternary composition spread. In this area, thermal conductivity values are close to the expected alloy limit (i.e., $10 \mathrm{~W} \mathrm{~m}^{-1} \mathrm{~K}^{-1}$ ). Besides, no simple correlation between thermal conductivity and composition maps can be visually established. Theoretical calculations based on first principle will be discussed in future works to find cross-correlation between the various ternary alloys.

\section{CONCLUSION AND OUTLOOK}

In this paper, we have introduced an improved version of the HPTR setup: High-Throughput Thermoreflectance setup. We have demonstrated its capabilities to carry out multiple thermal conductivity measurements over large surface samples. Using two synchronized femtosecond oscillators significantly reduces the acquisition time of one single area measurement to few seconds. In addition of being artefact-free and time-saving, many improvements on the setup guarantee similar conditions over multiple measurements as well as superior precision by eliminating most of the human-induced errors. This setup has been used to study a ternary Fe-Si-Ge library deposited on a $100 \mathrm{~mm}$ diameter sapphire substrate and synthesized by wedge-type multilayer deposition method. First high-throughput measurements have highlighted crystalline and amorphous phases on the library. Amorphous phases with thermal conductivity close to $1.1 \mathrm{~W} \mathrm{~m}^{-1} \mathrm{~K}^{-1}$ seem to be related to the multi-wedge deposition method and the chosen material system. Other deposition methods like co-deposition on a hot substrate are currently investigated to improve crystallinity of the different ternary phases. Crystalline phases, with thermal conductivity values close to the alloy literature, have been found to be mostly located in the center of the ternary system, i.e., around FeSiGe. The coupling of HT-thermal conductivity measurements with HT-electrical conductivities, using four-point probe method, will finally reveal ternary composition with optimized 
transport properties and improved thermoelectric figure of merit.

\section{ACKNOWLEDGMENTS}

The authors would like to thank the M-Eranet 2014 ICETS Project (A.L., A.F. DFG Project No. LU1175/16-1). The authors would like to thank O. Bernard for his helpful contribution throughout the measurements.

${ }^{1}$ G. Pernot, M. Stoffel, I. Savic, F. Pezzoli, P. Chen, G. Savelli, A. Jacquot, J. Schumann, U. Denker, I. Mönch, C. Deneke, O. G. Schmidt, J. M. Rampnoux, S. Wang, M. Plissonnier, A. Rastelli, S. Dilhaire, and N. Mingo, Nat. Mater. 9, 491 (2010).

${ }^{2}$ B. Vermeersch, A. M. S. Mohammed, G. Pernot, Y. R. Koh, and A. Shakouri, Phys. Rev. B 91, 085203 (2015); e-print arXiv:1406.7342.

${ }^{3}$ C. A. Paddock and G. L. Eesley, J. Appl. Phys. 60, 285 (1986).

${ }^{4}$ C. A. Paddock and G. L. Eesley, Opt. Lett. 11, 273 (1986).

${ }^{5}$ S. Dilhaire, S. Grauby, W. Claeys, and J.-C. Batsale, Microelectron. J. 35, 811 (2004).

${ }^{6}$ R. J. Stevens, A. N. Smith, and P. M. Norris, J. Heat Transfer 127, 315 (2005).

${ }^{7}$ B. Vermeersch, G. Pernot, H. Lu, J.-H. Bahk, A. Gossard, and A. Shakouri, Phys. Rev. B 88, 214302 (2013).
${ }^{8}$ R. H. M. Groeneveld and R. Sprik, Phys. Rev. B 51, 11433 (1995).

${ }^{9}$ J. Chen, W.-K. Chen, J. Tang, and P. M. Rentzepis, Proc. Natl. Acad. Sci. U. S. A. 108, 18887 (2011).

${ }^{10}$ O. B. Wright, J. Appl. Phys. 71, 1617 (1992)

${ }^{11}$ D. G. Cahill, K. Goodson, and A. Majumdar, J. Heat Transfer 124, 223 (2002).

${ }^{12}$ D. G. Cahill, Rev. Sci. Instrum. 75, 5119 (2004).

${ }^{13}$ S. Dilhaire, G. Pernot, G. Calbris, J. M. Rampnoux, and S. Grauby, J. Appl. Phys. 110, 114314 (2011).

${ }^{14}$ P. A. Elzinga, R. J. Kneisler, F. E. Lytle, Y. Jiang, G. B. King, and N. M. Laurendeau, Appl. Opt. 26, 4303 (1987).

${ }^{15}$ X. Zheng, D. G. Cahill, P. Krasnochtchekov, R. Averback, and J.-C. Zhao, Acta Mater. 55, 5177 (2007).

${ }^{16}$ A. J. Schmidt, X. Chen, and G. Chen, Rev. Sci. Instrum. 79, 114902 (2008).

${ }^{17}$ M. Fedorov, J. Thermoelectr. 51-60 (2009), ISSN 1607-8829.

${ }^{18}$ Y. Niwa, Y. Todaka, T. Masuda, T. Kawai, and M. Umemoto, Mater. Trans. 50, 1725 (2009).

${ }^{19}$ K. Mars, H. Ihou-Mouko, G. Pont, J. Tobola, and H. Scherrer, J. Electron. Mater. 38, 1360 (2009).

${ }^{20}$ M. I. Fedorov and G. N. Isachenko, Jpn. J. Appl. Phys., Part 1 54, 07JA05 (2015).

${ }^{21}$ A. Ludwig, R. Zarnetta, S. Hamann, A. Savan, and S. Thienhaus, Int. J. Mater. Res. 99, 1144 (2008).

${ }^{22}$ A. Janghorban, J. Pfetzing-Micklich, J. Frenzel, and A. Ludwig, Adv. Eng. Mater. 16, 588 (2014) 